\title{
A KOROVKIN TYPE APPROXIMATION THEOREM FOR SET-VALUED FUNCTIONS
}

\author{
KLAUS KEIMEL AND WALTER ROTH
}

(Communicated by John B. Conway)

\begin{abstract}
This paper is a contribution to the problem of approximating continuous functions $F$ defined on a compact Hausdorff space $X$, where the value $F(x)$ is a compact convex set in $\mathbf{R}^{n}$ for every $x$ in $X$. More specifically we show how to transfer Korovkin type approximation theorems for real-valued continuous functions to this set-valued situation.
\end{abstract}

1. Introduction. We consider the following problem: Given a convex body in the plane or in space whose shape and position is continuously varying in time (see Figure 1). Think of a convex body growing continuously. We measure its shape at discrete time instances. The problem is, whether one can approximate the growth function arbitrarily well by interpolating the shapes measured at sufficiently many instances.

Mathematically the growth function is modelled by a multivalued function $F$ associating a compact convex subset $F(x)$ of $\mathbf{R}^{n}$ to every value $x \in[0,1]$. We need a couple of special functions (see Figure 2): For a given $K \in \operatorname{Conv}\left(\mathbf{R}^{n}\right)$, the set of compact convex subsets of $\mathbf{R}^{n}, K$ will denote the constant function $F(x)=K$, while $x \mathbf{B}$ and $x^{2} \mathbf{B}$ denote the functions $F(x)=x \mathbf{B}$ and $F(x)=x^{2} \mathbf{B}$, where $\mathbf{B}$ is the euclidean unit ball in $\mathbf{R}^{n}$.

Recall that $\operatorname{Conv}\left(\mathbf{R}^{n}\right)$ is a convex cone with the usual addition and scalar multiplication for subsets of a linear space, and the Hausdorff metric on $\operatorname{Conv}\left(\mathbf{R}^{n}\right)$ is defined by

$$
d(K, L)=\inf \{\varepsilon>0 \mid K \subset L+\varepsilon \mathbf{B} \text { and } L \subset K+\varepsilon \mathbf{B}\} \quad \text { for } K, L \in \operatorname{Conv}\left(\mathbf{R}^{n}\right) .
$$

The growth function $F$ is supposed to be continuous with respect to this metric. If we know $F(x)$ for $x=j / n, j=0,1, \ldots, n$, we may interpolate by the $n$th Bernstein polynomial $B_{n}(F)$ associated with $F$ :

$$
B_{n}(F)=\sum_{j=0}^{n}\left(\begin{array}{l}
n \\
j
\end{array}\right) F\left(\frac{j}{n}\right) x^{j}(1-x)^{n-j}, \quad 0 \leq x \leq 1 .
$$

R. A. Vitale [9] has proved the following

THEOREM 1.1. For every continuous function $F:[0,1] \rightarrow \operatorname{Conv}\left(\mathbf{R}^{n}\right)$ the sequence $B_{n}(F)$ of Bernstein polynomials converges to $F$ uniformly in $x$.

For this result Vitale gives two proofs. Firstly, he gives a direct proof. Secondly, he derives this theorem from a general Korovkin type approximation theorem for

Received by the editors August 31, 1987 and, in revised form, November 16, 1987.

1980 Mathematics Subject Classification (1985 Revision). Primary 47B38, 47B55; Secondary 41A36, 41A63. 


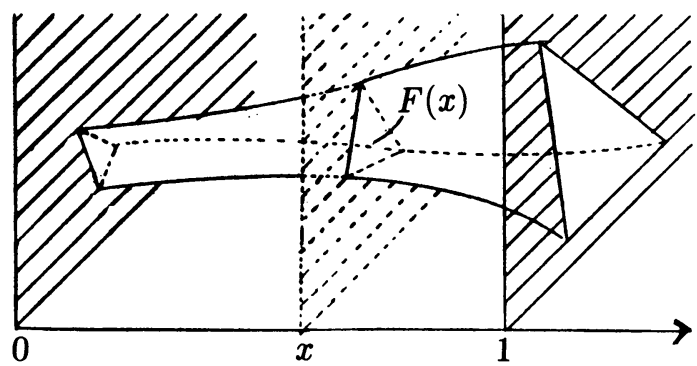

FIGURE 1

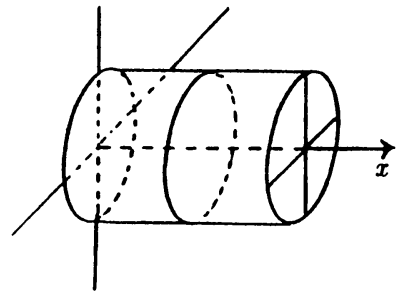

$F(x)=K$

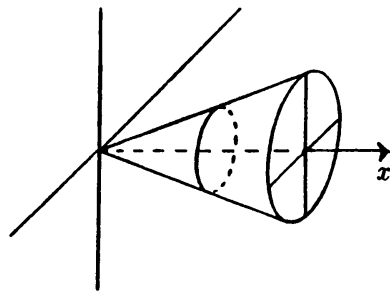

$F(x)=x \mathbf{B}$

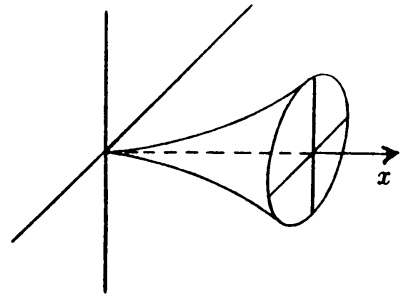

$F(x)=x^{2} \mathbf{B}$

\section{FIGURE 2}

set-valued functions which can be viewed as a general scheme for proving approximation theorems for set-valued functions.

Vitale's proof is rather long and technical. A. Jung [7] has found a shorter proof based on order theoretical arguments in the context of the theory of continuous lattices. We want to show in this note how set-valued Korovkin type approximation theorems can be derived directly from well-known Korovkin theorems for real-valued functions. At the same time, our approach yields a considerable generalization of Vitale's result.

2. Korovkin type theorems for real-valued functions. Let $X$ be a compact Hausdorff space. By $C(X)$ we denote the Banach space of real-valued continuous functions on $X$. We consider a set $M \subset C(X)$ of "test functions", and we denote by $\operatorname{span}(M)$ the linear subspace of $C(X)$ spanned by $M$.

The Korovkin closure $K(M)$ is defined to be the set of all functions $f \in C(X)$ which satisfy the following property:

For every equicontinuous net $\left(T_{\alpha}\right)$ of positive linear operators on $C(X)$ one has:

If $T_{\alpha}(g) \rightarrow g$ for all $g \in M$, then $T_{\alpha}(f) \rightarrow f$.

One says that $M$ is a Korovkin system for $C(X)$ if $K(M)=C(X)$, i.e. if (*) is fulfilled for all $f \in C(X)$.

Note. (a) If the constant function 1 belongs to $M$, then the hypothesis of equicontinuity in (*) is superfluous. Indeed, if $T_{\alpha}(1) \rightarrow 1$ then the net $\left(T_{\alpha}\right)$ of positive linear operators is automatically equicontinuous. 
(b) In many papers, condition $(*)$ is restricted to sequences $\left(T_{n}\right)$ of positive linear operators instead of nets. For compact metric spaces $X$, this is equivalent to the above.

We shall use the following two classical examples of Korovkin systems:

EXAMPLES. (a) On the unit interval $X=[0,1]$ the polynomials

$$
p_{0}(x)=1, \quad p_{1}(x)=x, \quad p_{2}(x)=x^{2}
$$

form a Korovkin system.

(b) On the euclidean unit sphere $S^{n-1}$ in $\mathbf{R}^{n}$, the coordinate projections

$$
p_{i}\left(x_{1}, \ldots, x_{n}\right)=x_{i} \quad(i=1, \ldots, n)
$$

together with the constant function 1 form a Korovkin system.

The following theorem does not seem to be in the literature in this explicite form although it can be derived from more general results as in [4 or 6]. In the case that $M$ contains the constant function 1 , it is due to H. Bauer [1] and H. Berens and G. G. Lorentz [3].

THEOREM 2.1. For any family $M$ of continuous functions on a compact Hausdorff space $X$ and any $f \in C(X)$ the following conditions are equivalent:

(i) $f$ belongs to the Korovkin closure of $M$.

(ii) $f(x)=\sup _{\varepsilon>0} \inf \{g(x) \mid g \in \operatorname{span}(M)$ and $g \geq f-\varepsilon\}$

$$
=\inf _{\varepsilon>0} \sup \{g(x) \mid g \in \operatorname{span}(M) \text { and } g \leq f+\varepsilon\} \text { for all } x \in X \text {. }
$$

(iii) For every $x \in X$ and every positive Radon measure $\mu$ on $X$ one has $\mu(f)=$ $f(x)$ provided that $\mu(g)=g(x)$ for all $g \in M$.

In particular $M$ is a Korovkin system for $C(X)$ if and only if (ii), or equivalently (iii), is satisfied for all $f \in C(X)$.

For a corollary let $X$ and $Y$ be compact Hausdorff spaces. There are natural embeddings of $C(X)$ and $C(Y)$ into $C(X \times Y)$; indeed, every function $f: X \rightarrow \mathbf{R}$ may be considered as a function from $X \times Y$ into $\mathbf{R}$ not depending on the second variable and, likewise, for functions on $Y$. With this convention in mind we may state

COROLLARY 2.2. If $M_{1}$ is a Korovkin system for $C(X)$ and $M_{2}$ for $C(Y)$, then $M=M_{1} \cup M_{2}$ is a Korovkin system for $C(X \times Y)$.

Proof. Consider $(x, y) \in X \times Y$ and suppose that $\mu$ is a positive measure on $X \times Y$ such that $\mu(g)=g(x, y)$ for all $g \in M$, i.e. $\mu(g)=g(x)$ for all $g \in M_{1}$, and $\mu(g)=g(y)$ for all $g \in M_{2}$. As $M_{1}$ and $M_{2}$ are Korovkin systems for $C(X)$ and $C(Y)$, we conclude that $\mu(f)=f(x)$ for all $f \in C(X)$ and $\mu(f)=f(y)$ for all $f \in C(Y)$. Thus, the support of $\mu$ is contained in $\{x\} \times Y$ as well as in $X \times\{y\}$. So $\mu=\lambda \varepsilon_{(x, y)}$; clearly $\lambda=1$, and consequently $\mu=\varepsilon_{(x, y)}$. Thus condition (iii) of Theorem 2.1 is satisfied for all $f \in C(X \times Y)$.

3. Korovkin type theorems for set-valued functions. We now return to the situation of $\S 1$. Instead of the unit interval we consider an arbitrary compact Hausdorff space $X$, and we denote by

$$
\mathscr{C}=C\left(X, \operatorname{Conv}\left(\mathbf{R}^{n}\right)\right)
$$

the set of all continuous functions $F$ defined on $X$ with values $F(x)$ in the set $\operatorname{Conv}\left(\mathbf{R}^{n}\right)$ of compact convex subsets of $\mathbf{R}^{n}$. Continuity is understood with respect 
to the Hausdorff metric on $\operatorname{Conv}\left(\mathbf{R}^{n}\right)$. B denotes the unit ball for an arbitrary norm on $\mathbf{R}^{n}$. As in $\S 1$, for functions $F, G \in \mathscr{C}$ we define

an addition by $(F+G)(x)=F(x)+G(x)$,

a scalar multiplication by $(\lambda F)(x)=\lambda F(x)$ for $\lambda \geq 0$,

an order by $F \leq G$ iff $F(x) \subset G(x)$ for all $x$, and we consider on $\mathscr{C}$ the topology of uniform convergence.

An operator $T: \mathscr{C} \rightarrow \mathscr{C}$ is called linear if

$$
T(F+G)=T(F)+T(G) \text { and } T(\lambda F)=\lambda T(F)
$$

for all $F, G \in \mathscr{C}$ and all $\lambda \geq 0$. It is called monotone if

$$
F \leq G \text { implies } T(F) \leq T(G) .
$$

As in the real-valued case, we shall say that a set $\mathscr{M} \subset \mathscr{C}$ of text functions is a Korovkin system for $\mathscr{C}$ if the following holds:

For every equicontinuous net $\left(T_{\alpha}\right)$ of monotone linear operators on

(**) $\mathscr{C}$ one has:

If $T_{\alpha}(G) \rightarrow G$ for all $G \in \mathscr{M}$, then $T_{\alpha}(F) \rightarrow F$ for all $F \in \mathscr{C}$.

Note. (a) If the constant function $\mathbf{B}$ belongs to $\mathscr{M}$, then the equicontinuity of the net $\left(T_{\alpha}\right)$ already follows from $T_{\alpha}(\mathbf{B}) \rightarrow \mathbf{B}$.

(b) For compact metric spaces $X$, one may restrict to sequences $\left(T_{n}\right)$ instead of nets $\left(T_{\alpha}\right)$ of operators in definition $(* *)$.

The following theorem which is our main result allows to transfer Korovkin systems from the single-valued to the multivalued case:

THEOREM 3.1. Let $X$ be a compact Hausdorff space, and $\mathbf{B}$ the unit ball for an arbitrary norm on $\mathbf{R}^{n}$. If $M$ is a Korovkin system of nonnegative functions for $C(X)$, then

$$
\mathscr{M}=\{x \rightarrow f(x) \mathbf{B} \mid f \in M\} \cup\{\text { all constant functions }\}
$$

is a Korovkin system for $\mathscr{C}$.

Proof. We denote by $Y$ the dual unit sphere of $\mathbf{B}$, i.e. the set of all linear functionals $y$ on $\mathbf{R}^{n}$ such that

$$
\|y\|=\sup \{y(x) \mid x \in \mathbf{B}\}=1 .
$$

Topologically $Y$ is homeomorphic to the euclidean sphere $S^{n-1}$.

With every compact convex set $C \subset \mathbf{R}^{n}$ we associate the classical support functional

$$
p_{C}: Y \rightarrow \mathbf{R}
$$

defined by $p_{C}(y)=\sup \{y(x) \mid x \in C\}$. The following facts are well known (see e.g. [8]) and easy to check:

(a) Since $p_{C}$ is sublinear on $\mathbf{R}^{n}$ it is continuous, thus $p_{C} \in C(Y)$.

(b) $p_{\mathbf{B}}(y)=1$ for all $y \in Y$.

For two compact convex sets $C$ and $D$ one clearly has:

(c) $p_{C+D}=p_{C}+p_{D}$ and $p_{\lambda C}=\lambda p_{C}$ for all $\lambda \geq 0$.

(d) $\sup \left(p_{C}, p_{D}\right)=p_{E}$, where $E$ is the convex hull of $C \cup D$.

(e) $C \subset D+\varepsilon \mathbf{B}$ if and only if $p_{C} \leq p_{D}+\varepsilon$, in particular $C \subset D$ iff $p_{C} \leq p_{D}$. 
(f) The Hausdorff metric on $\operatorname{Conv}\left(\mathbf{R}^{n}\right)$ corresponds with the supremum norm on $C(Y)$, since

$$
d(C, D)=\left\|p_{C}-p_{D}\right\|
$$

We conclude that $C \rightarrow p_{C}$ is a linear isometric order embedding of $\operatorname{Conv}\left(\mathbf{R}^{n}\right)$ into $C(Y)$. The linear subspace $L=\left\{p_{C}-p_{D} \mid C, D \in \operatorname{Conv}\left(\mathbf{R}^{n}\right)\right\}$ is a vector lattice by (d), containing 1 by (b). As clearly $L$ separates the points, $L$ is dense in $C(Y)$ by the Stone-Weierstrass Theorem.

The embedding $p$ : $\operatorname{Conv}\left(\mathbf{R}^{n}\right) \rightarrow C(Y)$ yields a linear isometric order embedding

$$
i: \mathscr{C} \rightarrow C(X, C(Y))
$$

given by $i(F)(x)=p_{F(x)}$ for all $F \in \mathscr{C}$ and all $x \in X$. Combining with the classical isomorphism

$$
j: C(X, C(Y)) \rightarrow C(X \times Y)
$$

given by $j(f)(x, y)=(f(x))(y)$ for all $f \in C(X, C(Y))$ and all $(x, y) \in X \times Y$, we obtain a linear order embedding

$$
k: \mathscr{C} \rightarrow C(X \times Y)
$$

Again the image of $k$ generates a dense vector sublattice of $C(X \times Y)$ and contains the constant function 1 . Thus, every monotone linear operator $T$ on $\mathscr{C}$, extends uniquely to a positive linear operator $\bar{T}$ on $C(X \times Y)$. And for an equicontinuous family $\left(T_{\alpha}\right)$ of monotone linear operators on $\mathscr{C}$ the family $\left(\bar{T}_{\alpha}\right)$ of extensions is equicontinuous on $C(X \times Y)$.

Thus, for Theorem 3.1 , it just remains to prove that $k(\mathscr{K})$ is a Korovkin system for $C(X \times Y)$. One easily verifies that, under $k$,

$$
x \rightarrow f(x) \text { B goes to }(x, y) \rightarrow f(x),
$$

the constant function

$$
x \rightarrow K \text { goes to }(x, y) \rightarrow p_{K}(y) .
$$

As $M$ is a Korovkin system for $X$ and the functions $p_{C}$ for $C \in \operatorname{Conv}\left(\mathbf{R}^{n}\right)$ generate a dense linear subspace of $C(Y)$, Corollary 2.2 allows us to conclude that $k(\mathscr{M})$ is a Korovkin system for $C(X \times Y)$, and the proof of Theorem 3.1 is complete.

In general, it is not necessary to include all constant functions in $\mathscr{M}$. It suffices to take a family $K_{1}, \ldots, K_{n}$ of compact convex sets such that the corresponding functions $p_{K_{1}}, \ldots, p_{K_{n}}$ form a Korovkin system for $C(Y)$. For example if $\mathbf{B}$ is the euclidean unit ball in $\mathbf{R}^{n}$, then $Y$ is the euclidean unit sphere $S^{n-1}$. For $S^{n-1}$ the coordinate projections

$$
p_{i}:\left(x_{1}, \ldots, x_{n}\right) \rightarrow x_{i}
$$

for $i=1, \ldots, n$ together with the constant function 1 form a Korovkin system. As $p_{\mathrm{B}}=1$ and $p_{e_{i}}=p_{i}$, where $e_{i}=\{(0, \ldots, 1, \ldots, 0)\}$ is the singleton set having the $i$ th canonical basis vector as its only member, we conclude

THEOREM 3.2. Let $X$ be a compact Hausdorff space, and $\mathbf{B}$ the euclidean unit ball in $\mathbf{R}^{n}$. If $M$ is a Korovkin system of nonnegative functions for $C(X)$, then the functions

$$
x \rightarrow f(x) \mathbf{B}, \quad f \in M,
$$


together with the constant functions with values

$$
\mathbf{B}, e_{1}, \ldots, e_{n}, \text { respectively, }
$$

form a Korovkin system for $\mathscr{C}$.

EXAMPLES. (a) If we use the Korovkin system $\left\{1, x, x^{2}\right\}$ on $X=[0,1]$, we immediately obtain Vitale's result [9], that the constant functions together with $x \rightarrow x \mathbf{B}$ and $x \rightarrow x^{2} \mathbf{B}$ constitute a Korovkin system for $C\left([0,1], \operatorname{Conv}\left(\mathbf{R}^{n}\right)\right)$.

(b) For $X=S^{1}$ (the unit circle) we identify functions on $S^{1}$ with periodic functions on $\mathbf{R}$ and we obtain: For the $2 \pi$-periodic continuous functions $F: \mathbf{R} \rightarrow$ $\operatorname{Conv}\left(\mathbf{R}^{n}\right)$ the functions

$$
\sin t \cdot \mathbf{B}, \cos t \cdot \mathbf{B}
$$

together with the constant functions with values

$$
\mathbf{B}, e_{1}, \ldots, e_{n} \text {, respectively, }
$$

constitute a Korovkin system.

\section{REFERENCES}

1. H. Bauer, Theorems of Korovkin type for adapted spaces, Ann. Inst. Fourier (Grenoble) 23 (1973), 245-260.

2. _ Approximationssätze und abstrakte Ränder, Math. Phys. Sem. Berichte 13 (1976), 141173.

3. H. Berens and G. G. Lorentz, Theorems of Korovkin type for positive linear operators on Banach lattices, Approximation Theory (G. G. Lorentz, ed.), Academic Press, New York, 1973.

4. K. Donner, Extension of positive operators and Korovkin theorems, Lecture Notes in Math., Vol. 904, Springer-Verlag, Berlin, Heidelberg and New York, 1982.

5. H. O. Flösser, Korovkinsche Approximation stetiger Funktionen, Jahrbuch Überblicke Mathematik, 1980, Bibliographisches Institut Mannheim-Wien-Zürich, pp. 93-119.

6. H. O. Flösser, R. Irmisch and W. Roth, Infimum-stable convex cones and approximation, Proc. London Math. Soc. (3) 42 (1981), 104-120.

7. A. Jung, Stetige Verbände und Approximationssätze, Diplomarbeit, Darmstadt, 1983.

8. H. Minkowski, Theorie der konvexen Körper, insbesondere Begründung ihres Oberflächenbegriffs, Ges. Abhandlungen, Vol. 2, Leipzig, Berlin, 1911, pp. 131-229.

9. R. A. Vitale, Approximation of convex set-valued functions, J. Approx. Theory 26 (1979), 301-316.

FaChbereich Mathematik, Technische Hochschule, D-6100 DaRmstadt, West GERMANY

Department of MAthematics, University of Bahrain, Manama, Bahrain 